\title{
Vénéreau-type polynomials as potential counterexamples
}

\author{
Drew Lewis* \\ Washington University in St. Louis \\ andrew@math.wustl.edu
}

November 6, 2018

\begin{abstract}
We study some properties of the Vénéreau polynomials $b_{m}=y+x^{m}\left(x z+y\left(y u+z^{2}\right)\right) \in \mathbb{C}[x, y, z, u]$, a sequence of proposed counterexamples to the Abhyankar-Sathaye embedding conjecture and the DolgachevWeisfeiler conjecture. It is well known that these are hyperplanes and residual coordinates, and for $m \geq 3$, they are $\mathbb{C}[x]$-coordinates. For $m=1,2$, it is only known that they are 1 -stable $\mathbb{C}[x]$-coordinates. We show that $b_{2}$ is in fact a $\mathbb{C}[x]$-coordinate. We introduce the notion of Vénéreau-type polynomials, and show that these are all hyperplanes and residual coordinates. We show that some of these Vénéreau-type polynomials are in fact $\mathbb{C}[x]$-coordinates; the rest remain potential counterexamples to the aforementioned conjectures. For those that we show to be coordinates, we also show that any automorphism with one of them as a component is stably tame. The remainder are stably tame, 1 -stable $\mathbb{C}[x]$-coordinates.
\end{abstract}

\section{Introduction}

Let $A$ (and all other rings) be a commutative ring with one throughout. An $A$-coordinate (if $A$ is understood, we simply say coordinate; some authors prefer the term variable) is a polynomial $f \in A^{[n]}$ for which there exist $f_{2}, \ldots f_{n} \in A^{[n]}$ such that $A\left[f, f_{2}, \ldots, f_{n}\right]=A^{[n]}$. It is natural to ask when a polynomial is a coordinate; this question is extremely deep and has been studied for some time. There are several conjectures concerning the identification of coordinates. First, the well-known Embedding Conjecture asserts, in short, that hyperplanes are coordinates:

Conjecture 1. (Abhyankar-Sathaye) Let $A$ be $a \mathbb{Q}$-algebra and $f \in A^{[n]}$. If $A^{[n]} /(f) \cong A^{[n-1]}$, then $f$ is a coordinate of $A^{[n]}$.

This is known only for $n=2$ with $A$ a polynomial ring over a field (11, 13], [11). We remark that the condition of $A$ being a $\mathbb{Q}$-algebra is necessary, even with $n=2$.

If we restrict our attention to $A=\mathbb{C}^{[r]}$, a special case of the Dolgachev-Weisfeiler Conjecture asserts that residual coordinates are coordinates:

Conjecture 2. Suppose $A=\mathbb{C}^{[r]}$, and let $f \in A^{[n]}$. If $A[f] \hookrightarrow A^{[n]}$ is an affine fibration, then $f$ is an A-coordinate.

In studying these kinds of problems, Vénéreau [16] and Berson [3] independently wrote down a polynomial in four variables, now known as the Vénéreau polynomial, that satisfies the hypotheses of these two conjectures (with $A=\mathbb{C}[x]$ ). It is often given as the first polynomial in the sequence

$$
b_{m}:=y+x^{m}\left(x z+y\left(y u+z^{2}\right)\right)
$$

Vénéreau was able to show that $b_{m}$ is a coordinate for $m \geq 3$, but the $m=1$ and $m=2$ cases proved elusive. Subsequently, many coordinate-like properties were shown about $b_{1}$ and $b_{2}$ ( e.g. [15], 6], [8]).

\footnotetext{
*Current affiliation: University of Alabama, amlewis@as.ua.edu
} 
We show that the second Vénéreau polynomial $b_{2}$ is a coordinate. We go on to highlight a key difference between $b_{1}$ and $b_{2}$. In the process, we describe a more general class of polynomials we term Vénéreautype polynomials. These are all hyperplanes and strongly residual coordinates; we show many of them to be coordinates, but are left with a class of potential counterexamples (including $b_{1}$ ) to the above conjectures. We go on to show that every coordinate-like property known to us to hold for $b_{1}$ also holds for any Vénéreau-type polynomial.

\section{Preliminaries}

For a ring $A$, we will adopt the standard notation of using $\mathrm{GA}_{n}(A)$ to denote the general automorphism group $\operatorname{Aut}_{\operatorname{Spec} A} \operatorname{Spec} A^{[n]}$, and $\mathrm{MA}_{n}(A)$ for the endomorphism group $\operatorname{End}_{\operatorname{Spec} A} \operatorname{Spec} A^{[n]}$. Note that $\operatorname{GA}_{n}(A)$ is naturally anti-isomorphic to $\operatorname{Aut}_{A} A^{[n]}$ (in fact, some authors choose to define it as such). $\mathrm{EA}_{n}(A)$ denotes the elementary group generated by automorphisms fixing $n-1$ variables, and $\mathrm{TA}_{n}(A):=\left\langle\mathrm{EA}_{n}(A), \mathrm{GL}_{n}(A)\right\rangle$ is called the tame subgroup. Given $\phi \in \mathrm{GA}_{n}(A)$, we will use $J \phi$ for the Jacobian determinant. We also require the following definitions:

Definition 1. Let $A$ be a ring and $f \in A^{[n]}$.

1. $f$ is a hyperplane if $A^{[n]} /(f) \cong A^{[n-1]}$.

2. $f$ is a residual coordinate if $A[f] \hookrightarrow A^{[n]}$ is an affine fibration; that is, if $A[f] \hookrightarrow A^{[n]}$ is flat and $A^{[n]} \otimes_{A[f]} \kappa(\mathfrak{p}) \cong \kappa(\mathfrak{p})^{[n-1]}$ for all $\mathfrak{p} \in \operatorname{Spec} A[f]$.

3. Let $x \in A \backslash A^{*}$ be a non-zero divisor. $f$ is a strongly $x$-residual coordinate if $\bar{f}$ is a coordinate in $(A / x A)^{[n]}$ and $f$ is a coordinate in the localization $\left(A_{x}\right)^{[n]}$. If $x$ is clear from context, we may simply say strongly residual coordinate.

4. $f$ is an $r$-stable coordinate (or just stable coordinate) if $f$ is a coordinate in $A^{[n+r]}$ for some $r \in \mathbb{N}$.

We are primarily interested in the case $A=\mathbb{C}^{[r]}$, in which case one easily checks that strongly residual coordinates are indeed residual coordinates. As observed in [5], a result of Asanuma [2] combined with the Quillen-Suslin Theorem yields

Theorem 1. Let $A=\mathbb{C}^{[r]}$ for some $r \in \mathbb{N}$. Then every residual coordinate (and hence, every strongly residual coordinate) is a stable coordinate.

A recent result in this same sprit that we will find useful is due to Berson, van den Essen, and Wright (a special case of Theorem 4.5 from [4])

Theorem 2. Let $\phi \in \mathrm{GA}_{n}(\mathbb{C}[x])$ with $J \phi=1$. If $\phi \in \mathrm{TA}_{n}\left(\mathbb{C}\left[x, x^{-1}\right]\right)$, and $\bar{\phi} \in \mathrm{EA}_{n}(\mathbb{C})$ (where $\bar{\phi}$ denotes the image modulo $x)$, then $\phi$ is stably tame.

We also make use of the following well known result, a special case of the overring principle (14] Proposition 1.1.7, for example):

Theorem 3. Let $A$ be a reduced ring and $x \in A$ a non-zero divisor. Let $\phi \in \operatorname{MA}_{n}(A) \subset \mathrm{MA}_{n}\left(A_{x}\right)$ with $J \phi \in A^{*}$. Then $\phi \in \mathrm{GA}_{n}(A)$ if and only if $\phi \in \mathrm{GA}_{n}\left(A_{x}\right)$

One can produce many exotic objects from this kind of construction. The famous Nagata map, perhaps the simplest example of a wild (i.e. not tame) automorphism, arises in this manner as an element of $\mathrm{GA}_{2}(\mathbb{C}[x]) \subset \mathrm{GA}_{3}(\mathbb{C}):$

$$
\begin{aligned}
\sigma & =\left(x, y+x\left(x z-y^{2}\right), z+2 y\left(x z-y^{2}\right)+x\left(x z-y^{2}\right)^{2}\right) \\
& =\left(x, y, z+\frac{y^{2}}{x}\right) \circ\left(x, y+x^{2} z, z\right) \circ\left(x, y, z-\frac{y^{2}}{x}\right)
\end{aligned}
$$


We use this approach to construct a variety of strongly residual coordinates. Consider $\alpha \in \mathrm{GA}_{n}\left(\mathbb{C}\left[x, x^{-1}\right][y]\right)$, and let $Q \in \mathbb{C}[x]^{[n]}=\mathbb{C}[x]\left[z_{1}, \ldots, z_{n}\right]$ such that $\alpha(Q) \in \mathbb{C}[x, y]^{[n]}$. Then $f:=y+x \alpha(Q)$ is a strongly $x$ residual coordinate; indeed, $\bar{f} \equiv y(\bmod x)$ is trivially a coordinate; and over $\mathbb{C}\left[x, x^{-1}\right]$, we have $\phi(y)=f$ where $\phi \in \mathrm{GA}_{n+1}\left(\mathbb{C}\left[x, x^{-1}\right]\right)$ is given by

$$
\phi=\left(y+x Q, z_{1}, \ldots, z_{n}\right) \circ \alpha
$$

Conjecture 2 asserts that these should all be coordinates. If $Q \in x^{m} \mathbb{C}[x]^{[n]}$ for a sufficiently large $m$, then we quickly see that $f$ is in fact a coordinate.

Theorem 4. Let $\alpha=\left(f_{1}, \ldots, f_{n}\right) \in \mathrm{GA}_{n}\left(\mathbb{C}\left[x, x^{-1}\right][y]\right)$ and $Q \in \mathbb{C}[x]^{[n]}$. Then for all $m>0, y+$ $x^{m} Q\left(f_{1}, \ldots, f_{n}\right)$ is a $\mathbb{C}[x]$-coordinate. To be precise, write $\alpha^{-1}=\left(g_{1}, \ldots, g_{n}\right)$ and define, for $k>0$ and $1 \leq j \leq n$,

$$
q_{j, k}=\min \left\{q \mid x^{q k} \frac{\partial^{k} g_{j}}{\partial y^{k}}\left(y, f_{1}, \ldots, f_{n}\right) \in \mathbb{C}[x, y]^{[n]}\right\}
$$

Also define

$$
\begin{aligned}
m_{1} & :=\max _{1 \leq j \leq n ; 0<k}\left\{q_{j, k}\right\} \\
m_{2} & :=\min \left\{m \in \mathbb{N} \mid x^{m} Q\left(f_{1}, \ldots, f_{n}\right) \in \mathbb{C}[x, y]^{[n]}\right\}
\end{aligned}
$$

Then $y+x^{m} Q\left(f_{1}, \ldots, f_{n}\right)$ is a coordinate if $m \geq m_{1}+m_{2}$.

Proof. This is just an application of Taylor's formula. Let

$$
\phi=\alpha^{-1} \circ\left(y+x^{m} Q, z_{1}, \ldots, z_{n}\right) \circ \alpha
$$

and compute

$$
\begin{aligned}
\phi\left(z_{j}\right) & =g_{j}\left(y+x^{m} Q\left(f_{1}, \ldots, f_{m}\right), f_{1}, \ldots, f_{n}\right) \\
& =\sum_{k=0} \frac{1}{k !} \frac{\partial^{k} g_{j}}{\partial y^{k}}\left(y, f_{1}, \ldots, f_{n}\right)\left(x^{m} Q\left(f_{1}, \ldots, f_{n}\right)\right)^{k} \\
& =z_{j}+\sum_{k=1} \frac{1}{k !} x^{k\left(m-m_{2}-m_{1}\right)}\left(x^{k m_{1}} \frac{\partial^{k} g_{j}}{\partial y^{k}}\left(y, f_{1}, \ldots, f_{n}\right)\right)\left(x^{m_{2}} Q\left(f_{1}, \ldots, f_{n}\right)\right)^{k}
\end{aligned}
$$

It is now immediate from (44) and (5) that $\phi\left(z_{j}\right) \in \mathbb{C}[x, y]^{[n]}$. One quickly checks that $J \phi=1$, so by Theorem 3. $\phi \in \mathrm{GA}_{n}(\mathbb{C}[x])$.

\section{The Vénéreau polynomials}

We start by defining a derivation on $\mathbb{C}[y, z, u]$ by

$$
D:=y \frac{\partial}{\partial z}-2 z \frac{\partial}{\partial u}
$$

$D$ is triangular and thus locally nilpotent. We also define

$$
p:=y u+z^{2} \quad v:=x z+y p \quad w:=x^{2} u-2 x z p-y p^{2}
$$

Note that $\operatorname{ker} D=\mathbb{C}[y, p]$, and $\exp (p D) \in \mathrm{GA}_{3}(\mathbb{C})$ is (up to a change of variables) the Nagata map (2). By defining $D x=0, D$ naturally extends to a derivation on $\mathbb{C}[x]^{[3]}$ and thus $\mathbb{C}\left[x, x^{-1}\right]^{[3]}$ with kernel $\mathbb{C}\left[x, x^{-1}\right][y, p]$. Thus we may consider $\psi:=\exp \left(\frac{p}{x} D\right) \in \mathrm{GA}_{3}\left(\mathbb{C}\left[x, x^{-1}\right]\right)$. One quickly computes

$$
\psi=\left(y, \frac{v}{x}, \frac{w}{x^{2}}\right)
$$


We note that since $\psi$ fixes $p$, we quickly obtain the relation

$$
y w+v^{2}=x^{2} p
$$

As in (3), we thus see the Vénéreau polynomials naturally as the $y$-component of

$$
\left(y+x^{m+1} z, z, u\right) \circ \psi
$$

Applying Theorem 4 in this case recovers Vénéreau's result that $b_{m}$ is a coordinate for $m \geq 3$. A slight variation of that technique shows that $b_{2}$ is a coordinate.

Theorem 5. The second Vénéreau polynomial $b_{2}=y+x^{2}\left(x z+y\left(y u+z^{2}\right)\right)$ is a coordinate.

Abbreviated Proof. Define $\varphi_{m} \in \mathrm{GA}_{3}\left(\mathbb{C}\left[x, x^{-1}\right]\right)$ by

$$
\varphi_{m}=\psi^{-1} \circ\left(y, z-\frac{1}{2} x^{m+1} u, u\right) \circ\left(y+x^{m+1} z, z, u\right) \circ \psi
$$

One quickly checks that $\varphi_{m}(y)=b_{m}$ and $J \varphi_{m}=1$; so by Theorem 3, all that is left to check is that for $m \geq 2, \varphi_{m} \in \mathrm{MA}_{3}(\mathbb{C}[x])$. This is straightforward to check and is shown in more generality below in Theorem 13

Remark 1. We can also see $\varphi_{m}$ as an exponential: namely, let $d$ be the jacobian derivation given by $d=$ $J\left(p+\frac{1}{2} x^{m-2} v w, w, \cdot\right)$. One can check that $d$ is locally nilpotent and $\varphi_{m}=\exp \left(\frac{1}{2} x^{m-1} d\right)$.

Prior to our promised discussion of Vénéreau-type polynomials, we would like to highlight a significant difference between $b_{1}$ and $b_{2}$. For this, we define:

Notation 2. Let $\alpha \in \mathrm{GA}_{n}\left(\mathbb{C}\left[x, x^{-1}\right]\right)$. Define

$$
\alpha^{*}\left(\mathrm{GA}_{n}(\mathbb{C}[x])\right)=\left\{\alpha^{-1} \circ \phi \circ \alpha \mid \phi \in \mathrm{GA}_{n}(\mathbb{C}[x])\right\} \subset \mathrm{GA}_{n}\left(\mathbb{C}\left[x, x^{-1}\right]\right)
$$

The proof of Theorem 5 shows that $b_{2}$ is a coordinate of $\varphi_{2} \in \psi^{*}\left(\mathrm{GA}_{3}(\mathbb{C}[x])\right)$. More strongly, defining $\tilde{\psi} \in \mathrm{GA}_{3}\left(\mathbb{C}\left[x, x^{-1}\right]\right)$ by

$$
\tilde{\psi}:=(y, v, w)=\left(y, x z, x^{2} u\right) \circ \psi
$$

we may even write

$$
\varphi_{m}=\tilde{\psi} \circ\left(y, z-\frac{1}{2} x^{m} u, u\right) \circ\left(y+x^{m} z, z, u\right) \circ \tilde{\psi}
$$

and thus see $\varphi_{2} \in \tilde{\psi}^{*}\left(\mathrm{GA}_{3}(\mathbb{C}[x])\right)$. However, this is too much to ask of $b_{1}$.

Theorem 6. There is no automorphism $\phi \in \mathrm{GA}_{3}(\mathbb{C}[x]) \cap \tilde{\psi}^{*}\left(\mathrm{GA}_{3}(\mathbb{C}[x])\right)$ with $\phi(y)=b_{1}$.

This follows from the following technical result, whose proof we momentarily defer:

Theorem 7. Let $\alpha \in \mathrm{GA}_{3}(\mathbb{C}[x])$.

1. If $\tilde{\psi}^{-1} \circ \alpha \circ \tilde{\psi} \in \mathrm{GA}_{3}(\mathbb{C}[x])$, then $\alpha(p) \in\left(p, x^{2}\right)$.

2. Suppose that $\alpha(y) \equiv y(\bmod x)$. Then $\tilde{\psi}^{-1} \circ \alpha \circ \tilde{\psi} \in \mathrm{GA}_{3}(\mathbb{C}[x])$ if and only if $\alpha(p)=p+x^{2} F+x p A+x^{3} B$ for some $A, B \in \mathbb{C}[x]^{[3]}$ and $F \in \mathbb{C}[y, p]$ such that $\alpha \equiv \exp (F D)(\bmod x)\left(\right.$ recall $D=y \frac{\partial}{\partial z}-2 z \frac{\partial}{\partial u}$ from (6)).

3. Suppose that $\alpha(y) \equiv y(\bmod x), \phi=\tilde{\psi}^{-1} \circ \alpha \circ \tilde{\psi} \in \mathrm{GA}_{3}(\mathbb{C}[x])$, and $\phi(p) \equiv p(\bmod x)$. If $\alpha$ is stably tame over $\mathbb{C}[x]$, then $\phi$ is stably tame over $\mathbb{C}[x]$ as well.

Proof of Theorem [6. Suppose $\phi \in \mathrm{GA}_{3}(\mathbb{C}[x]) \cap \tilde{\psi}^{*}\left(\mathrm{GA}_{3}(\mathbb{C}[x])\right)$ and $\phi(y)=b_{1}$. Then $\phi=\tilde{\psi}^{-1} \circ \alpha \circ \tilde{\psi}$ for some $\alpha \in \mathrm{GA}_{3}(\mathbb{C}[x])$. In particular, since $\phi(y)=b_{1}$, we may write

$$
\alpha=\left(y+x z, \sum_{i=0} x^{i} G_{i}, \sum_{i=0} x^{i} H_{i}\right)
$$


for some $G_{i}, H_{i} \in \mathbb{C}^{[3]}$. By Theorem $7(2)$, we have $\alpha \equiv \exp (F D)(\bmod x)$ for some $F \in \mathbb{C}[y, p]$. Thus

$$
G_{0}=z+y F \quad H_{0}=u-2 z F-y F^{2}
$$

Write $\alpha(p)=\sum_{i=0} x^{i} P_{i}$ for some $P_{i} \in \mathbb{C}^{[3]}$. On the one hand, we can compute directly from (13)

$$
\begin{aligned}
& P_{0}=y H_{0}+G_{0}^{2} \\
& P_{1}=y H_{1}+z H_{0}+2 G_{0} G_{1} \\
& P_{2}=y H_{2}+z H_{1}+2 G_{0} G_{2}+G_{1}^{2}
\end{aligned}
$$

On the other hand, from Theorem $7(2)$, we must have $P_{0}=p, P_{1} \in(p)$, and $P_{2} \equiv F(\bmod p)$. We will use these to derive a contradiction.

Claim 8. $G_{1} \equiv-\frac{1}{2} u(\bmod y, z)$.

Proof. Since $P_{1} \equiv 0(\bmod p)$, we apply (15) and compute

$$
\begin{aligned}
0 & \equiv y H_{1}+z H_{0}+2 G_{0} G_{1} & & (\bmod p) \\
& \equiv y H_{1}+z\left(u-2 z F-y F^{2}\right)+2(z-y F) G_{1} & & (\bmod p)
\end{aligned}
$$

with the second line following from (14). Noting that $p=y u+z^{2} \in\left(y, z^{2}\right)$, we may go modulo $\left(y, z^{2}\right)$ and obtain $0 \equiv z\left(u+2 G_{1}\right)\left(\bmod y, z^{2}\right)$, hence $G_{1} \equiv-\frac{1}{2} u(\bmod y, z)$.

Now, since $P_{2} \equiv F(\bmod p)$, we apply (16) and compute

$$
F \equiv y H_{2}+z H_{1}+2(z+y F) G_{2}+G_{1}^{2} \quad(\bmod p)
$$

Similar to above, since $p \in(y, z)$, we see $F \equiv G_{1}^{2}(\bmod y, z)$. Applying Claim 8 , we then have

$$
F \equiv \frac{1}{4} u^{2} \quad(\bmod y, z)
$$

However, $F \in \mathbb{C}[y, p]$, so $F \equiv a(\bmod y, z)$ for some $a \in \mathbb{C}$, a contradiction.

So while $b_{1}$ cannot be a coordinate of an automorphism of $\mathrm{GA}_{3}(\mathbb{C}[x]) \cap \tilde{\psi}^{*}\left(\mathrm{GA}_{3}(\mathbb{C}[x])\right)$, we pose the following:

Conjecture 3. $b_{1}$ is a coordinate if and only if it is a coordinate of an automorphism $\phi \in \mathrm{GA}_{3}(\mathbb{C}[x]) \cap$ $\psi^{*}\left(\mathrm{GA}_{3}(\mathbb{C}[x])\right)$.

We devote the rest of this section to proving Theorem 7 . First, we require a couple lemmas.

Lemma 9. $\mathbb{C}[y, v, w] \cap(x) \mathbb{C}[x][y, z, u]=\mathbb{C}[y, v, w] \cap\left(x^{2}\right) \mathbb{C}[x][y, z, u]=\left(y w+v^{2}\right) \mathbb{C}[y, v, w]$

Proof. Clearly the relation $y w+v^{2}=x^{2} p$ (from (9) ) guarantees

$$
\mathbb{C}[y, v, w] \cap x \mathbb{C}[x][y, z, u] \supset \mathbb{C}[y, v, w] \cap x^{2} \mathbb{C}[x][y, z, u] \supset\left(y w+v^{2}\right) \mathbb{C}[y, v, w]
$$

So we simply need to see $\mathbb{C}[y, v, w] \cap x \mathbb{C}[x][y, z, u] \subset\left(y w+v^{2}\right) \mathbb{C}[y, v, w]$. Note that we have a map $\alpha$ : $\mathbb{C}[y, v, w] \rightarrow \mathbb{C}\left[y, y p,-y p^{2}\right]$ obtained from going $\bmod x$. Clearly $y w+v^{2}$ is in the kernel of this map, so it descends to the quotient. Observing that $\mathbb{C}[y, v, w] /\left(y w+v^{2}\right) \cong \mathbb{C}\left[y, v, \frac{-v^{2}}{y}\right]$, we have the following commutative diagram:

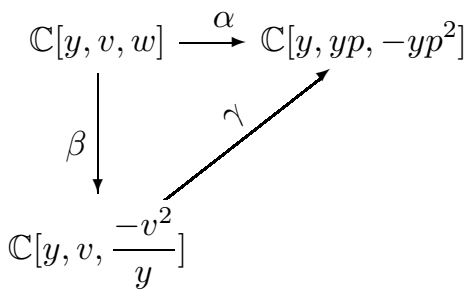


Note that $\gamma$ is in fact an isomorphism; hence $\operatorname{ker} \beta=\operatorname{ker} \alpha$. But $\beta$ is the quotient map, so ker $\beta=\left(y w+v^{2}\right)$, and $\operatorname{ker} \alpha=\mathbb{C}[y, v, w] \cap(x) \mathbb{C}[x][y, z, u]$.

Corollary 10. $\tilde{\psi}^{-1}\left(x^{2} \mathbb{C}[x][y, z, u]\right) \cap \mathbb{C}[x][y, z, u]=\left(x^{2}, p\right) \mathbb{C}[x][y, z, u]$

Proof. Applying $\tilde{\psi}$ throughout, this is equivalent to showing

$$
x^{2} \mathbb{C}[x][y, z, u] \cap \mathbb{C}[x][y, v, w]=\left(x^{2}, y w+v^{2}\right) \mathbb{C}[x][y, v, w]
$$

That the right side is contained in the left is immediate (recall from (9) that $y w+v^{2} \in x^{2} \mathbb{C}[x][y, z, u]$ ). For the opposite containment, suppose $A \in x^{2} \mathbb{C}[x][y, z, u] \cap \mathbb{C}[x][y, v, w]$ and write $A=\sum_{i=0} x^{i} A_{i}$ for some $A_{i} \in \mathbb{C}[y, v, w]$. It suffices to assume that $A=A_{0}+x A_{1}$. Since $A \in x^{2} \mathbb{C}[x][y, z, u] \subset x \mathbb{C}[x][y, z, u]$, and (trivially) $x A_{1} \in x \mathbb{C}[x][y, z, u]$, we must also have $A_{0} \in x \mathbb{C}[x][y, z, u]$. So $A_{0} \in x \mathbb{C}[x][y, z, u] \cap \mathbb{C}[y, v, w]=$ $\left(y w+v^{2}\right) \mathbb{C}[y, v, w]$ by Lemma 9 . Thus, we may now assume $A=x A_{1}$. Since $A \in x^{2} \mathbb{C}[x][y, z, u]$, we see $A_{1} \in x \mathbb{C}[x][y, z, u]$, and again applying Lemma 9 yields $A_{1} \in\left(y w+v^{2}\right) \mathbb{C}[y, v, w]$.

Lemma 11. Suppose $\phi \in \mathrm{GA}_{3}(\mathbb{C})$ with $\phi(y)=y$ and $\phi(p) \in(p)$. Then $\phi=\left(y, c z, c^{2} u\right) \circ \exp (P D)$ for some $c \in \mathbb{C}^{*}, P \in \mathbb{C}[y, p]$.

Proof. Write $\phi=\left(y, F_{1}, F_{2}\right)$ for some $F_{1}, F_{2} \in \mathbb{C}^{[3]}$. Since $\phi(p) \in(p)$ and $p$ is irreducible, we see $y F_{2}+F_{1}=r p$ for some $r \in \mathbb{C}^{*}$. Set $c=\frac{1}{r} J \phi \in \mathbb{C}^{*}$. Now we compute

$$
c y=\frac{1}{r} y J\left(y, F_{1}, F_{2}\right)=\frac{1}{r} J\left(y, F_{1}, y F_{2}+F_{1}^{2}\right)=J\left(y, F_{1}, p\right)
$$

Observe that $J(y, \cdot, p)=D$ (recall from (6) $\left.D=y \frac{\partial}{\partial z}-2 z \frac{\partial}{\partial u}\right)$, so we may rewrite this as $D\left(F_{1}\right)=c y \in \operatorname{ker} D$; thus $F_{1}=c z+\tilde{P}$ for some $\tilde{P} \in \operatorname{ker} D=\mathbb{C}[y, p]$. We now recompute

$$
r p=\phi(p)=y F_{2}+(c z+\tilde{P})^{2}
$$

Comparing the $z^{2}$ terms on each side, we deduce $r=c^{2}$. We also must have $y \mid \tilde{P}$. Set $P=\frac{\tilde{P}}{c y} \in \mathbb{C}[y, p]$. Plugging this back in to the relation $\phi(p)=r p=c^{2} p$, we obtain

$$
c^{2} y u=y F_{2}+2 c^{2} y z P+c^{2} y^{2} P^{2}
$$

Thus $F_{2}=c^{2}\left(u-2 z P-y P^{2}\right)$, and we have $\phi=\left(y, c(z+y P), c^{2}\left(u-2 z P-y P^{2}\right)\right)=\left(y, c z, c^{2} u\right) \circ \exp (P D)$.

We now have the required tools, and may proceed with the proof of Theorem 7 ,

Proof of Theorem 7 . Define $\phi=\tilde{\psi}^{-1} \circ \alpha \circ \tilde{\psi}$. For (1), suppose $\phi \in \mathrm{GA}_{3}(\mathbb{C}[x])$; in particular, $\phi(p) \in \mathbb{C}[x]^{[3]}$. We thus compute, noting that (12) and (9) imply $\tilde{\psi}^{-1}(p)=\frac{p}{x^{2}}$,

$$
\begin{aligned}
\left(\tilde{\psi}^{-1} \circ \alpha \circ \tilde{\psi}\right)(p) & \in \mathbb{C}[x][y, z, u] \\
(\alpha \circ \tilde{\psi})\left(\frac{p}{x^{2}}\right) & \in \mathbb{C}[x][y, z, u] \\
\alpha\left(\frac{p}{x^{2}}\right) & \in \tilde{\psi}^{-1}(\mathbb{C}[x][y, z, u]) \\
\alpha(p) & \in \tilde{\psi}^{-1}\left(x^{2} \mathbb{C}[x][y, z, u]\right)
\end{aligned}
$$

Since $\alpha \in \mathrm{GA}_{3}(\mathbb{C}[x])$, we thus have $\alpha(p) \in \tilde{\psi}^{-1}\left(x^{2} \mathbb{C}[x][y, z, u]\right) \cap \mathbb{C}[x][y, z, u]$. Applying Corollary 10, we thus have $\alpha(p) \in\left(x^{2}, p\right) \mathbb{C}[x][y, z, u]$, establishing assertion (1) of the theorem.

We now assume for the remainder that $\alpha(y) \equiv y(\bmod x)$. Write

$$
\alpha=\left(y+x Q, \sum_{i=0} x^{i} G_{i}, \sum_{i=0} x^{i} H_{i}\right)
$$


for some $Q \in \mathbb{C}[x]^{[3]}, G_{i}, H_{i} \in \mathbb{C}^{[3]}$. Let

$$
Q=\sum_{i=0} x^{i} Q_{i} \quad \alpha(p)=\sum_{i=0} x^{i} P_{i}
$$

for some $Q_{i}, P_{i} \in \mathbb{C}^{[3]}$. Direct computation shows

$$
P_{0}=y H_{0}+G_{0}^{2} \quad P_{1}=y H_{1}+Q_{0} H_{0}+2 G_{0} G_{1}
$$
(17),

For one direction of $(2)$, assume $\phi \in \mathrm{GA}_{3}(\mathbb{C}[x])$. In particular, $\phi(p) \in \mathbb{C}[x]^{[3]}$. We then compute, using

$$
\phi\left(x^{2} p\right)=\left(\tilde{\psi}^{-1} \circ \alpha \circ \tilde{\psi}\right)\left(x^{2} p\right)=(\alpha \circ \tilde{\psi})(p)=\tilde{\psi}\left(\sum_{i=0} x^{i} P_{i}\right)
$$

Since $\phi\left(x^{2} p\right) \in x^{2} \mathbb{C}[x]^{[3]}$, we thus see $P_{0}+x P_{1} \in \tilde{\psi}^{-1}\left(x^{2} \mathbb{C}[x]^{[3]}\right) \cap \mathbb{C}[x]^{[3]}=\left(x^{2}, p\right) \mathbb{C}[x]^{[3]}$ by Corollary 10. Since $P_{0}, P_{1} \in \mathbb{C}^{[3]}$, we then see $P_{0}+x P_{1} \in(p) \mathbb{C}[x]^{[3]}$, whence $P_{0}, P_{1} \in(p) \mathbb{C}^{[3]}$.

Consider $\bar{\alpha}=\left(y, G_{0}, H_{0}\right) \in \mathrm{GA}_{3}(\mathbb{C})$, the image modulo $x$; Since $p$ is irreducible and $\bar{\alpha}(p)=P_{0} \in(p)$, by Lemma $11 \bar{\alpha}=\left(y, c z, c^{2} u\right) \circ \exp (F D)$ for some $c \in \mathbb{C}^{*}, F \in \mathbb{C}[y, p]$. Thus we have

$$
G_{0}=c(z+y F) \quad H_{0}=c^{2}\left(u-2 z F-y F^{2}\right)
$$

In particular, we see from (18) that we must have $P_{0}=c^{2} p$. Since $\alpha(p) \in\left(p, x^{2}\right)$ by part (1) and $P_{1} \in(p) C^{[3]}$, we can write $\alpha(p)=c^{2} p+x p A^{\prime}+x^{2} B^{\prime}$ for some $A^{\prime}, B^{\prime} \in \mathbb{C}[x]^{[3]}$. Now,

$$
\begin{aligned}
\phi(x z) & =\phi(v-y p) \\
& =\left(\tilde{\psi}^{-1} \circ \alpha \circ \tilde{\psi}\right)(v-y p) \\
& =(\alpha \circ \tilde{\psi})\left(z-y \frac{p}{x^{2}}\right) \\
& =\tilde{\psi}\left(\sum_{i=0} x^{i} G_{i}-(y+x Q)\left(\frac{p}{x^{2}}\left(c^{2}+x A^{\prime}\right)+B^{\prime}\right)\right) \\
& =\sum_{i=0} x^{i} \tilde{\psi}\left(G_{i}\right)-(y+x \tilde{\psi}(Q))\left(p\left(c^{2}+x \tilde{\psi}\left(A^{\prime}\right)\right)+\tilde{\psi}\left(B^{\prime}\right)\right)
\end{aligned}
$$

with the first equality arising from (7). Since $\phi(z) \in \mathbb{C}[x]^{[3]}$, we must have $\phi(x z) \in x \mathbb{C}[x]^{[3]}$, and thus

$$
\begin{aligned}
0 & \equiv \tilde{\psi}\left(G_{0}\right)-y\left(p c^{2}\right)-y \tilde{\psi}\left(B^{\prime}\right) & & (\bmod x) \\
0 & \equiv \tilde{\psi}(c(z+y F))-y\left(p c^{2}\right)-y \tilde{\psi}\left(B^{\prime}\right) & & (\bmod x) \\
0 & \equiv c(v-y p c)+y \tilde{\psi}\left(c F-B^{\prime}\right) & & (\bmod x) \\
0 & \equiv c y p(1-c)+y \tilde{\psi}\left(c F-B^{\prime}\right) & & (\bmod x)
\end{aligned}
$$

Observing that $\tilde{\psi}\left((y, z, u) \mathbb{C}[x]^{[3]}\right) \subset(x, y) \mathbb{C}[x]^{[3]}$ (coming from the fact that $y, v, w \in(x, y) \mathbb{C}[x]^{[3]}$ ), we must have $c=1$. We also must have $F-B^{\prime} \in \mathbb{C}[x]^{[3]} \cap \tilde{\psi}^{-1}\left(x \mathbb{C}[x]^{[3]}\right)=(x, p) \mathbb{C}[x]^{[3]}$ (from Corollary[10). Thus we write $B^{\prime}=F+x B+p A^{\prime \prime}$ for some $B \in \mathbb{C}[x]^{[3]}$. Setting $A=A^{\prime}+x A^{\prime \prime}$ gives $\alpha(p)=p+x^{2} F+x p A+x^{3} B$ as required. have

For the converse, assume $\alpha(p)=p+x^{2} F+x p A+x^{3} B$ and $\bar{\alpha} \equiv \exp (F D)(\bmod x)$. In particular, we

$$
G_{0}=z+y F \quad H_{0}=u-2 z F-y F^{2}
$$

Since $J \phi=J \alpha=J \bar{\alpha}=1$, it suffices to check that $\phi \in \mathrm{MA}_{3}(\mathbb{C}[x])$; compute

$$
\phi(y)=\left(\tilde{\psi}^{-1} \circ \alpha \circ \tilde{\psi}\right)(y)=(\alpha \circ \tilde{\psi})(y)=y+x Q(y, v, w) \in \mathbb{C}[x]^{[3]}
$$


Next, we show $\phi(x z) \in x \mathbb{C}[x]^{[3]}$. Again, using (7),

$$
\begin{aligned}
\phi(x z) & =\left(\tilde{\psi}^{-1} \circ \alpha \circ \tilde{\psi}\right)(v-y p) \\
& =(\alpha \circ \tilde{\psi})\left(z-y \frac{p}{x^{2}}\right) \\
& =\tilde{\psi}\left(\sum_{i=0} x^{i} G_{i}-(y+x Q)\left(\frac{1}{x^{2}}\right)\left(p+x^{2} F+x p A+x^{3} B\right)\right) \\
& =\sum_{i=0} x^{i} \tilde{\psi}\left(G_{i}\right)-(y+x \tilde{\psi}(Q))(p+\tilde{\psi}(F)+x(p \tilde{\psi}(A)+x \tilde{\psi}(B)))
\end{aligned}
$$

So $\phi(x z) \in \mathbb{C}[x]^{[3]}$. Going modulo $x$, and recalling from (19) that $G_{0}=z+y F$ (and thus $\tilde{\psi}\left(G_{0}\right)=v+y \tilde{\psi}(F)$ ), we obtain

$$
\phi(x z) \equiv(v+y \tilde{\psi}(F))-y(p+\tilde{\psi}(F)) \equiv 0 \quad(\bmod x)
$$

So we have $\phi(x z) \in x \mathbb{C}[x]^{[3]}$ and thus $\phi(z) \in \mathbb{C}[x]^{[3]}$. Since $\phi(y) \notin x \mathbb{C}[x]^{[3]}$, it suffices to check that $\phi(p) \in \mathbb{C}[x]^{[3]}$ as well (as then $\phi(u) \in \mathbb{C}[x]^{[3]}$ ).

$$
\begin{aligned}
\phi(p) & =\left(\tilde{\psi}^{-1} \circ \alpha \circ \tilde{\psi}\right)(p) \\
& =(\alpha \circ \tilde{\psi})\left(\frac{p}{x^{2}}\right) \\
& =\tilde{\psi}\left(\frac{1}{x^{2}}\left(p+x^{2} F+x p A+x^{3} B\right)\right) \\
& =p+\tilde{\psi}(F)+x(p \tilde{\psi}(A)+\tilde{\psi}(B))
\end{aligned}
$$

So $\phi(p) \in \mathbb{C}[x]^{[3]}$, and thus $\phi \in \mathrm{GA}_{3}\left(\mathbb{C}\left[x, x^{-1}\right]\right) \cap \mathrm{MA}_{3}(\mathbb{C}[x])$. Since $J \phi=J \alpha=1$, by Theorem $3 \phi \in$ $\mathrm{GA}_{3}(\mathbb{C}[x])$.

For the final part, we will use Theorem 2 and the following well known result of Smith [12]:

Theorem 12 (Smith). Let $A$ be a $\mathbb{Q}$-algebra and $D$ be a triangular derivation of $A^{[n]}$. Then for any $P \in \operatorname{ker} D$, $\exp (P D)$ is 1-stably tame.

Suppose $\phi, \alpha$ are as in (3). While $\phi \notin \mathrm{TA}_{3}\left(\mathbb{C}\left[x, x^{-1}\right]\right)$, Smith's theorem shows that $\tilde{\psi}$ is 1 -stably tame (over $\mathbb{C}\left[x, x^{-1}\right]$ ), hence $\phi$ is stably tame over $\mathbb{C}\left[x, x^{-1}\right]$. One also quickly checks that $J \phi=J \alpha \in \mathbb{C}^{*}$. So we only need to see that $\bar{\phi}$ is stably a composition of elementaries. By Lemma 11, since $\bar{\phi}(y)=y$ and $\bar{\phi}(p)=p$, a composition with a diagonal map allows us to assume $\bar{\phi}(p)=\exp (P D)$ for some $P \in \mathbb{C}[y, p]$ (and thus $J \phi=J \bar{\phi}=1)$. Again appealing to Smith's theorem, we thus have $\bar{\phi}(p) \in \mathrm{EA}_{4}(\mathbb{C})$. Thus by Theorem 2, $\phi$ is stably tame.

\section{Vénéreau-type Polynomials}

Instead of only considering the Vénéreau polynomials, one may generalize these slightly and still retain all the coordinate-like properties.

Definition 3. A Vénéreau-type polynomial is a polynomial of the form $y+x Q$ for some $Q \in \mathbb{C}[x][v, w]$.

Note that $Q=x^{m-1} v$ gives the Vénéreau polynomials $b_{m}$. We first give a sufficient condition for a Vénéreau-type polynomial to be a coordinate.

Theorem 13. If $Q=x^{2} Q_{1}+x v Q_{2}$ for some $Q_{1} \in \mathbb{C}[x][v, w]$ and $Q_{2} \in \mathbb{C}\left[v^{2}, w\right]$, then the Vénéreau-type polynomial $y+x Q$ is a coordinate.

Proof. Write $Q_{2}=\sum \alpha_{a, b} v^{2 a} w^{b}$ for some $\alpha_{a, b} \in \mathbb{C}$. Define $\alpha \in \mathrm{GA}_{3}(\mathbb{C}[x])$ by

$$
\alpha=\left(y+x Q(z, u), z-\frac{1}{2} x^{2} \sum \alpha_{a, b}(-1)^{a} y^{a} u^{a+b+1}, u\right)
$$


Direct computation shows

$$
\begin{aligned}
\alpha(p) & \equiv(y+x Q(z, u)) u+\left(z-\frac{1}{2} x^{2} \sum \alpha_{a, b}(-1)^{a} y^{a} u^{a+b+1}\right)^{2} & & \left(\bmod x^{3}\right) \\
& \equiv\left(y u+z^{2}\right)+x^{2} z\left(u Q_{2}(z, u)-\sum \alpha_{a, b}(-1)^{a} y^{a} u^{a+b+1}\right) & & \left(\bmod x^{3}\right) \\
& \equiv p+x^{2} z\left(\sum \alpha_{a, b} u^{b+1}\left(z^{2 a}-(-y u)^{a}\right)\right) & & \left(\bmod x^{3}\right)
\end{aligned}
$$

Noting that $z^{2 a}-(-y u)^{a} \in\left(z^{2}+y u\right)=(p)$, we thus have that $\alpha(p) \equiv p\left(\bmod \left(x p, x^{3}\right)\right)$. Thus, by Theorem $7(2), \phi:=\tilde{\psi}^{-1} \circ \alpha \circ \tilde{\psi} \in \mathrm{GA}_{3}(\mathbb{C}[x])$, and one quickly checks $\phi(y)=y+x Q$.

Corollary 14. The second Vénéreau polynomial $b_{2}$ is a coordinate.

It is interesting to note that the above automorphisms are stably tame; moreover, any automorphism of the above type, with a Vénéreau-type polynomial as a coordinate, must be stably tame.

Theorem 15. If $Q=x^{2} Q_{1}+x v Q_{2}$ for some $Q_{1} \in \mathbb{C}[x][v, w]$ and $Q_{2} \in \mathbb{C}\left[v^{2}, w\right]$, any $\varphi \in \mathrm{GA}_{3}(\mathbb{C}[x])$ with $\varphi(y)=y+x Q$ is stably tame.

Proof. First, note that the $\phi$ constructed in the proof of Theorem 13 is stably tame; indeed, the fact that $\alpha(p) \equiv p\left(\bmod \left(x p, x^{3}\right)\right)$ guarantees $\phi(p) \equiv p(\bmod x)$, thus $\phi$ is stably tame by part 3 of Theorem 7 Now, consider arbitrary $\varphi \in \mathrm{GA}_{3}(\mathbb{C}[x])$ with $\varphi(y)=y+x Q$. Then $\left(\varphi \circ \phi^{-1}\right)(y)=\left(\phi \circ \phi^{-1}\right)(y)=y$. In other words, $\varphi \circ \phi^{-1} \in \mathrm{GA}_{2}(\mathbb{C}[x][y])$. The main result of $[4$ states that any automorphism in two variables over a regular ring is stably tame; since $\mathbb{C}[x]$ is regular, so is $\mathbb{C}[x][y]$, thus the composition $\varphi \circ \phi^{-1}$ is stably tame. Since $\phi$ is also stably tame, we thus see that $\varphi$ is stably tame.

Among the Vénéreau-type polynomials, the Vénéreau complements are worth highlighting:

Definition 4. The polynomials $c_{m}:=y+x^{m} w(m \geq 1)$ are called Vénéreau complements

Theorem 16. The Vénéreau polynomial $b_{m}$ is a coordinate if and only if the Vénéreau complement $c_{2 m}$ is.

Proof. Define $\theta_{m} \in \mathrm{GA}_{3}\left(\mathbb{C}\left[x, x^{-1}\right]\right)$ by

$$
\theta_{m}=\tilde{\psi}^{-1} \circ\left(y+x^{m} z-\frac{1}{4} x^{2 m} u, z-\frac{1}{2} x^{m} u, u\right) \circ \tilde{\psi}
$$

One easily checks that $\theta_{m}(y)=b_{m}-\frac{1}{4} x^{2 m} w, \theta_{m}(w)=w$, and via Theorem[7, $\theta_{m} \in \mathrm{GA}_{3}(\mathbb{C}[x])$ for each $m \geq 1$. It thus follows that $b_{m}$ is a coordinate if and only if $y+\frac{1}{4} x^{2 m} w$ is, as $\left(y+\frac{1}{4} x^{2 m} w, *, *\right) \circ \theta_{m}=\left(b_{m}, *, *\right)$. Consider the automorphism $\beta_{m} \in \mathrm{GA}_{4}(\mathbb{C})$ given by $\beta_{m}=\left(\lambda x, y, \lambda z, \lambda^{2} u\right)$ where $\lambda^{2 m+4}=4$. One easily checks that $\beta_{m}(w)=\lambda^{4} w$ and thus $\beta_{m}\left(y+\frac{1}{4} x^{2 m} w\right)=c_{2 m}$. Thus conjugation by $\beta_{m}$ shows that $c_{2 m}$ is a coordinate if and only if $y+\frac{1}{4} x^{2 m} w$ is as well.

Note that by Theorem 13, $c_{m}$ is a coordinate for $m \geq 3$. So this slightly stronger fact gives an alternate proof of the fact that $b_{m}$ is a coordinate for all $m \geq 2$.

Remark 2. Theorem 16 can be generalized slightly: let $P(w) \in \mathbb{C}[x][w]$. Then $y+x v P(w)$ is a coordinate if and only if $y+x^{2} w P(w)^{2}$ is. The proof is almost identical.

The remainder is devoted to showing that all Vénéreau-type polynomials satisfy a variety of coordinatelike properties that are known to hold for the Vénéreau polynomial. In particular, we show that they are strongly $x$-residual coordinates (Proposition 17), hyperplanes (Theorem 20), hyperplane fibrations (Theorem 22), and stably tame 1-stable coordinates (Theorem 30).

Proposition 17. Let $f=y+x Q, Q \in \mathbb{C}[x][v, w]$ be a Vénéreau-type polynomial. Then $f$ is a strongly $x$-residual coordinate.

Proof. Since $Q \in \mathbb{C}[x][v, w], \phi=\tilde{\psi}^{-1} \circ(y+x Q(z, u), z, u) \circ \tilde{\psi} \in \mathrm{GA}_{3}\left(\mathbb{C}\left[x, x^{-1}\right]\right)$ and $\phi(y)=y+x Q$, so $y+x Q$ is an $\mathbb{C}\left[x, x^{-1}\right]$-coordinate. Clearly $\bar{f} \equiv y(\bmod x)$, so $f$ is a strongly $x$-residual coordinate. 
To see that Vénéreau-type polynomials are hyperplanes, we use the following fact (pointed out to me by Arno van den Essen), which also appears (with $b=0$ ) in [7]. A special case of this was used in [16 to show that $f_{1}$ is a hyperplane.

Lemma 18. Let $A$ be a commutative ring, $a, b \in A$, and $g \in A[y]$. Then $A[y] /(y+a g(y)-b) \cong A[y] /(y+$ $g(a y+b))$.

Proof. We compute below, where the first isomorphism is given by sending $y$ to $a y+b$; we then identify $t=-g(a y+b)$, and use this to rewrite the relation in terms of $t$ only.

$$
\begin{aligned}
A[y] /(y+a g(y)-b) & \cong A[a y+b] /(a(y+g(a y+b))) \\
& \cong A[a y+b, t] /(a(y+g(a y+b)), t+g(a y+b)) \\
& \cong A[a y+b, t] /(a(y-t), t+g(a y+b)) \\
& \cong A[t] /(t+g(a t+b))
\end{aligned}
$$

Corollary 19. Let $A$ be a commutative ring, $a, b \in A^{[n]}$ and $g \in A^{[n]}[y]$. Then $y+a g(y)-b$ is a hyperplane (over $A$ ) if and only if $y+g(a y+b)$ is as well.

Theorem 20. Let $f=y+x Q, Q \in \mathbb{C}[x][v, w]$, be a Vénéreau-type polynomial. Then $f$ is an $\mathbb{C}[x]$-hyperplane of $\mathbb{C}[x]^{[3]}$; that is, $\mathbb{C}[x]^{[3]} /(f) \cong \cong_{\mathbb{C}[x]} \mathbb{C}[x]^{[2]}$.

Proof. Similar to (7), define

$$
p_{0}=x y u+z^{2} \quad v_{0}=z+y p_{0} \quad w_{0}=x u-2 v_{0} p_{0}+y p_{0}^{2}
$$

Claim 21. It suffices to check that $y+x Q_{0}$ is a coordinate for any $Q_{0} \in \mathbb{C}[x]\left[v_{0}, w_{0}\right]$.

Proof. Applying Corollary 19 to $f$ (with $a=x$ and $b=0$ ) yields that $f$ is a hyperplane if and only if $f_{0}=y+Q\left(x v_{0}, x w_{0}\right)$ is. We can write $Q\left(x v_{0}, x w_{0}\right)=x Q_{0}+\lambda$ for some $Q_{0} \in \mathbb{C}[x]\left[v_{0}, w_{0}\right]$ and $\lambda \in \mathbb{C}$; hence $f_{0}=y+x Q_{0}+\lambda$. Thus, if $y+x Q_{0}$ is a coordinate, so is $f_{0}$, hence $f_{0}$ is a hyperplane and (by Corollary 19) so is $f$.

We have thus reduced the theorem to showing that $y+x Q_{0}$ is a coordinate for any $Q_{0} \in \mathbb{C}[x]\left[v_{0}, w_{0}\right]$. The proof of this is quite analagous to that of Theorem[5. First, define $D_{0}=x y \frac{\partial}{\partial z}-2 z \frac{\partial}{\partial u}$, and observe that $\operatorname{ker} D_{0}=\mathbb{C}[x]\left[y, p_{0}\right]$. Also define

$$
\psi_{0}=\exp \left(\frac{p_{0}}{x} D_{0}\right)=\left(y, v_{0}, \frac{w_{0}}{x}\right)
$$

In particular, since $\psi_{0}$ fixes $p_{0}$, we see

$$
y w_{0}+v_{0}^{2}=p_{0}
$$

Also define

$$
\alpha_{0}:=\left(y+x Q_{0}(z, u), z, u\right) \quad \phi_{0}:=\psi_{0}^{-1} \circ \alpha_{0} \circ \psi_{0}
$$

One immediately sees that $\phi_{0} \in \mathrm{GA}_{3}\left(\mathbb{C}\left[x, x^{-1}\right]\right), J \phi_{0}=1$, and $\phi_{0}(y)=y+x Q_{0}$. So by Theorem 3, we need only see that $\phi_{0} \in \mathrm{MA}_{3}(\mathbb{C}[x])$. We first compute $\phi_{0}\left(v_{0}\right), \phi_{0}\left(w_{0}\right)$, and $\phi_{0}\left(p_{0}\right)$, and use those to compute $\phi_{0}(z), \phi_{0}(u)$.

$$
\begin{aligned}
\phi_{0}\left(v_{0}\right) & =\left(\psi_{0}^{-1} \circ \alpha_{0} \circ \psi_{0}\right)\left(v_{0}\right) \\
& =\left(\alpha_{0} \circ \psi_{0}\right)(z) \\
& =\left(\psi_{0}\right)(z) \\
& =v_{0}
\end{aligned}
$$

$$
\begin{aligned}
\phi_{0}\left(w_{0}\right) & =\left(\psi_{0}^{-1} \circ \alpha_{0} \circ \psi_{0}\right)\left(w_{0}\right) \\
& =\left(\alpha_{0} \circ \psi_{0}\right)(x u) \\
& =\left(\psi_{0}\right)(x u) \\
& =w_{0}
\end{aligned}
$$

Now from (21) we see

$$
\phi_{0}\left(p_{0}\right)=\phi_{0}\left(y w_{0}+v_{0}^{2}\right)=\left(y+x Q_{0}\right) w_{0}+v_{0}^{2}=p_{0}+x Q_{0} w_{0}
$$


Finally, we compute, using (20)

$$
\begin{aligned}
\phi_{0}(z) & =\phi_{0}\left(v_{0}-y p_{0}\right) \\
& =v_{0}-\left(y+x Q_{0}\right)\left(p_{0}+x Q_{0} w_{0}\right)
\end{aligned}
$$

and

$$
\begin{aligned}
\phi_{0}(u)= & \frac{1}{x} \phi_{0}\left(w_{0}+2 v_{0} p_{0}-y p_{0}^{2}\right) \\
= & \frac{1}{x}\left(w_{0}+2 v_{0}\left(p_{0}+x Q_{0} w_{0}\right)-\left(y+x Q_{0}\right)\left(p_{0}+x Q_{0} w_{0}\right)^{2}\right) \\
= & \frac{1}{x}\left(w_{0}+2 v_{0} p_{0}-y p_{0}^{2}\right)+Q_{0}\left(2 w_{0} v_{0}-2 w_{0} y p_{0}-p_{0}^{2}\right)+ \\
& \quad-x Q_{0}^{2}\left(y w_{0}^{2}-2 w_{0} p_{0}\right)-x^{2} Q_{0}^{3} w_{0}^{2} \\
= & u+Q_{0}\left(2 w_{0} v_{0}-2 w_{0} y p_{0}-p_{0}^{2}\right)-x Q_{0}^{2}\left(y w_{0}^{2}-2 w_{0} p_{0}\right)-x^{2} Q_{0}^{3} w_{0}^{2}
\end{aligned}
$$

Thus $\phi_{0} \in \mathrm{MA}_{3}(\mathbb{C}[x])$ and hence $\phi_{0} \in \mathrm{GA}_{3}(\mathbb{C}[x])$.

We even have something stronger, namely that all Vénéreau-type polynomials define hyperplane fibrations. To show this for $b_{1}$, Vénéreau made use of the fact that $b_{1}=y+x\left(x z+y\left(y u+z^{2}\right)\right)$ is linear in $u$; this does not hold for Vénéreau-type polynomials in general, so we have to do a bit more work.

Theorem 22. Let $f=y+x Q, Q \in \mathbb{C}[x][v, w]$, be a Vénéreau-type polynomial. Then for each $c \in \mathbb{C}, f-c$ is an $\mathbb{C}[x]$-hyperplane of $\mathbb{C}[x]^{[3]}$.

Proof. By Theorem 20, we assume $c \neq 0$. As in the proof of that theorem, we would like to apply Corollary 19. However, now we must do so with $b=c($ still $a=x)$. Define

$$
p_{1}=(x y+c) u+z^{2} \quad v_{1}=x z+(x y+c) p_{1} \quad w_{1}=x^{2} u-2 v_{1} p_{1}+(x y+c) p_{1}^{2}
$$

Then Corollary 19 yields that we need only show $y+Q\left(v_{1}, w_{1}\right)$ is a hyperplane (in fact, we show it is a coordinate). The general idea is as follows: construct an automorphism $\left(y+Q\left(v_{1}, w_{1}\right), *, *\right) \in \mathrm{GA}_{3}\left(\mathbb{C}\left[x, x^{-1}\right]\right)$, then compose on the left with automorphisms fixing $y$ until it is also in $\mathrm{MA}_{3}\left(\mathbb{C}\left[x, x^{-1}\right]\right)$. Then we apply Theorem 3 . We start by defining a derivation over $\mathbb{C}\left[x, x^{-1}\right]$ by

$$
D_{1}=(x y+c) \frac{\partial}{\partial z}-2 z \frac{\partial}{\partial u}
$$

$D_{1}$ is triangular, and hence locally nilpotent. One easily checks that $\operatorname{ker} D_{1}=\mathbb{C}[x]\left[y, p_{1}\right]$. Define

$$
\phi_{0}=\exp \left(\frac{p_{1}}{x} D_{1}\right)=\left(y, \frac{v_{1}}{x}, \frac{w_{1}}{x^{2}}\right)
$$

Since $x^{2} p_{1} \in \operatorname{ker} D_{1}$, we must have $\phi_{0}\left(x^{2} p_{1}\right)=x^{2} p_{1}$ and thus obtain

$$
(x y+c) w_{1}+v_{1}^{2}=x^{2} p_{1}
$$

Our first step is to define

$$
\phi_{1}=\left(y+Q\left(x z, x^{2} u\right), z, u\right) \circ \phi_{0}=\left(y+Q\left(v_{1}, w_{1}\right), \frac{v_{1}}{x}, \frac{w_{1}}{x^{2}}\right)
$$

Next, we set

$$
\begin{aligned}
\phi_{2} & =\left(y, z, u+c^{-1} z^{2}\right) \circ \phi_{1} \\
& =\left(y+Q\left(v_{1}, w_{1}\right), \frac{v_{1}}{x}, \frac{c w_{1}+v_{1}^{2}}{c x^{2}}\right) \\
& =\left(y+Q\left(v_{1}, w_{1}\right), \frac{v_{1}}{x}, c^{-1} p_{1}-\frac{y w_{1}}{c x}\right)
\end{aligned}
$$

with the last equality following from (23). For the next step, we require the following: 
Claim 23. For any $G \in \mathbb{C}[x]^{[2]}, G\left(v_{1},-c^{-1} v_{1}^{2}\right) \equiv G\left(v_{1}, w_{1}\right)+x y G^{\prime}\left(v_{1}, w_{1}\right)\left(\bmod x^{2}\right)$ for some $G^{\prime} \in \mathbb{C}[x]^{[2]}$.

Proof. This is a straightforward computation, appealing to (23). Indeed, note that

$$
-c^{-1} v_{1}^{2}=w_{1}+c^{-1} x\left(y w_{1}-x p_{1}\right)
$$

We thus compute, applying Taylor's formula at the second step,

$$
\begin{aligned}
G\left(v_{1},-c^{-1} v_{1}^{2}\right) & \equiv G\left(v_{1}, w_{1}+c^{-1} x y w_{1}\right) & & \left(\bmod x^{2}\right) \\
& \equiv G\left(v_{1}, w_{1}\right)+c^{-1} x y w_{1} \frac{\partial G}{\partial w_{1}}\left(v_{1}, w_{1}\right) & & \left(\bmod x^{2}\right)
\end{aligned}
$$

Setting $G^{\prime}=c^{-1} w_{1} \frac{\partial G}{\partial w_{1}}\left(v_{1}, w_{1}\right)$ yields the claim.

Claim 24. For any $H \in \mathbb{C}[x]^{[3]}, G \in \mathbb{C}[x]^{[2]}$, there exists $H^{\prime} \in \mathbb{C}[x]^{[3]}$ such that

$$
H\left(y+G\left(v_{1}, w_{1}\right)-G\left(v_{1},-c^{-1} v_{1}^{2}\right), v_{1},-c^{-1} v_{1}^{2}\right) \equiv H\left(y, v_{1}, w_{1}\right)+x H^{\prime}\left(y, v_{1}, w_{1}\right) \quad\left(\bmod x^{2}\right)
$$

Moreover, if $x \mid H$, then $x \mid H^{\prime}$.

Proof. Let

$$
L:=H\left(y+G\left(v_{1}, w_{1}\right)-G\left(v_{1},-c^{-1} v_{1}^{2}\right), v_{1},-c^{-1} v_{1}^{2}\right)
$$

First, apply Claim 23 to $G$, obtaining $L=H\left(y+x y G^{\prime}\left(v_{1}, w_{1}\right)+x^{2} T_{1}, v_{1},-c^{-1} v_{1}^{2}\right)$ for some $T_{1} \in \mathbb{C}[x]^{[3]}$. Next, apply Taylor's formula in the first component, obtaining for some $H^{\prime} \in \mathbb{C}[x]\left[y, v_{1}, w_{1}\right]$ and $T_{2} \in \mathbb{C}[x]^{[3]}$,

$$
\begin{aligned}
L & =H\left(y, v_{1},-c^{-1} v_{1}^{2}\right)+x H^{\prime}\left(y, v_{1}, w_{1}\right)+x^{2} T_{2} \\
& =H\left(y, v_{1}, w_{1}+c^{-1} x y w_{1}-c^{-1} x^{2} p_{1}\right)+x H^{\prime}\left(y, v_{1}, w_{1}\right)+x^{2} T_{2}
\end{aligned}
$$

with the second equality following from (23). We now apply Taylor's formula in the third component, obtaining

$$
L \equiv H\left(y, v_{1}, w_{1}\right)+x H^{\prime \prime}\left(y, v_{1}, w_{1}\right) \quad\left(\bmod x^{2}\right)
$$

This is precisely the desired claim.

Claim 25. Suppose $\phi \in \mathrm{GA}_{3}\left(\mathbb{C}\left[x, x^{-1}\right]\right)$ is of the form

$$
\phi=\left(y+F\left(v_{1}, w_{1}\right), \frac{v_{1}}{x}, A+x^{r} B+x^{r-2} G\left(y, v_{1}, w_{1}\right)\right)
$$

for some $r \in \mathbb{Z}, A, B \in \mathbb{C}[x]^{[3]}, F \in \mathbb{C}[x]\left[v_{1}, w_{1}\right]$, and $G \in \mathbb{C}[x]\left[y, v_{1}, w_{1}\right]$. Then there exists $\tau \in$ $\mathrm{GA}_{3}\left(\mathbb{C}\left[x, x^{-1}\right]\right)$ such that $\tau \circ \phi=\left(y+F\left(v_{1}, w_{1}\right), \frac{v_{1}}{x}, A+x^{r} B^{\prime}\right)$ for some $B^{\prime} \in \mathbb{C}[x]^{[3]}$.

Proof. Define

$$
\tau=\left(y, z, u-x^{r-2} G\left(y-F\left(x z,-c^{-1}(x z)^{2}\right), x z,-c^{-1}(x z)^{2}\right)\right)
$$

Now compute

$$
\begin{aligned}
\tau \circ \phi= & \left(y+F\left(v_{1}, w_{1}\right), \frac{v_{1}}{x}, A+x^{r} B+\right. \\
& \left.x^{r-2}\left(G\left(y, v_{1}, w_{1}\right)-G\left(y+F\left(v_{1}, w_{1}\right)-F\left(v_{1},-c^{-1} v_{1}^{2}\right), v_{1},-c^{-1} v_{1}^{2}\right)\right)\right)
\end{aligned}
$$

Applying Claim 24, we obtain

$$
\tau \circ \phi=\left(y+F\left(v_{1}, w_{1}\right), \frac{v_{1}}{x}, A+x^{r} B^{\prime}+x^{r-1} G^{\prime}\left(y, v_{1}, w_{1}\right)\right)
$$

Note that, by Claim 24] if $x \mid G$, then $x \mid G^{\prime}$.

Now we can apply an induction step (replacing $B$ with $B^{\prime}$ and $G$ with $x G^{\prime}$ ) to obtain the desired result. 
Now, apply the preceding claim to $\phi_{2}(24)$ to obtain

$$
\phi_{3}:=\left(y+Q\left(v_{1}, w_{1}\right), \frac{v_{1}}{x}, c^{-1} p_{1}+x B\right)
$$

for some $B \in \mathbb{C}[x]^{[3]}$. Finally, set

$$
\begin{aligned}
\phi_{4} & =\left(y, z-\frac{c^{2} u}{x}, u\right) \circ \phi_{3} \\
& =\left(y+Q\left(v_{1}, w_{1}\right), \frac{v_{1}}{x}-\frac{c}{x}\left(p_{1}+x c B\right), c^{-1} p_{1}+x B\right) \\
& =\left(y+Q\left(v_{1}, w_{1}\right), z+y p_{1}-c^{2} B, c^{-1} p_{1}+x B\right)
\end{aligned}
$$

with the last equality coming from (22). Thus we have $\phi_{4} \in \mathrm{MA}_{3}(\mathbb{C}[x]) \cap \mathrm{GA}_{3}\left(\mathbb{C}\left[x, x^{-1}\right]\right)$, hence $\phi_{4} \in$ $\mathrm{GA}_{3}(\mathbb{C}[x])$ and $\phi_{4}(y)=y+Q\left(v_{1}, w_{1}\right)$, i.e. $y+Q\left(v_{1}, w_{1}\right)$ is a coordinate as required.

Before proceeding, we remark that Corollary 19 raises an interesting question:

Conjecture 4. Let $A$ be $a \mathbb{Q}$ algebra, $a, b \in A^{[n]}$, and $g \in A^{[n]}[y]$. Then $y+g(a y+b)$ is a coordinate if and only if $y+a g(y)-b$ is.

This is weaker than the Embedding Conjecture, but a proof of this conjecture would make every Vénéreautype polynomial a coordinate.

The next thing we prove is a generalization of a fact about $f_{1}$ from [15]. As described there, the motivation is the following well known lemma:

Lemma 26. Let $A$ be a ring, and $f \in A^{[n]}$. Then the following are equivalent

1. $f$ is a coordinate

2. $A^{[n]} \cong A[f] A[f]^{[n-1]}$

3. $A[c]^{[n]} /(f-c) \cong{ }_{A[c]} A[c]^{[n-1]}$, where $c$ is an additional indeterminate

In light of this, we may observe that

Corollary 27. Let $P(x, c) \in \mathbb{C}[x, c]$, and set $\tilde{R}=\mathbb{C}[x, c] /(P)$. Let $f$ be a Vénéreau-type polynomial. If $f$ is a coordinate of $\mathbb{C}[x][y, z, u]$, then $f-c$ is a $\tilde{R}$-hyperplane; i.e. $\tilde{R}[y, z, u] /(f-c) \cong_{\tilde{R}} \tilde{R}^{[2]}$.

So if we found one such $P$ for which a Vénéreau-type polynomial $f$ is not a $\tilde{R}$-hyperplane, then it could not be a coordinate.

Theorem 28. For any of the following polynomials $P_{i} \in \mathbb{C}[x, c]$ and any Vénéreau-type polynomial $f=$ $y+x Q, f-c$ is a $\tilde{R}_{i}$-hyperplane (where $\left.\tilde{R}_{i}:=\mathbb{C}[x, c] /\left(P_{i}\right)\right)$.

1. $P_{1}:=x-x_{0}\left(\right.$ where $\left.x_{0} \in \mathbb{C}\right)$

2. $P_{2}:=c-c_{0}\left(\right.$ where $\left.c_{0} \in \mathbb{C}\right)$

3. $P_{3}:=x^{2}-c^{3}$

Proof. For (1), first suppose $x_{0}=0$. Then $f-c \equiv y-c(\bmod x)$ is obviously a coordinate, hence a hyperplane. If $x_{0} \in \mathbb{C}^{*}$, then since $f-c$ is a $\mathbb{C}\left[x, x^{-1}\right]$ coordinate, $\bar{f}-c$ is a coordinate when we go $\bmod$ $x-x_{0}$, and hence is a hyperplane. For part $(2)$, note that $\mathbb{C}[x, c]^{[3]} /\left(c-c_{0}, f-c\right) \cong \mathbb{C}[x]^{[3]} /\left(f-c_{0}\right)$. But by Theorem 22 , we have $\mathbb{C}[x]^{[3]} /\left(f-c_{0}\right) \cong \mathbb{C}[x]^{[2]}$ giving the desired result.

For (3), we follow the approach sketched in [15] for the Vénéreau polynomials; this requires Corollary 19 and the following (Corollary 1.31 from [7), which is an application of the Abhyankar-Moh-Suzuki theorem:

Lemma 29. Let $\alpha(y, z, u) \in \mathbb{C}[t][y, z, u]$ be a $\mathbb{C}[t]$-coordinate, and let $\beta \in \mathbb{C}[t]$. If $\alpha(y, z, \beta u)$ is a $\mathbb{C}[t]$-residual coordinate, then it is also a $\mathbb{C}[t]$-coordinate, and hence $a \mathbb{C}[t]$-hyperplane. 
The key idea from [15] is that $\tilde{R}_{3} \cong \mathbb{C}\left[t^{2}, t^{3}\right]$, and using the main theorem from that paper, it suffices to show the image of $f-c$ in $\mathbb{C}\left[t^{2}, t^{3}\right]$ is a $\mathbb{C}[t]$-hyperplane. Note that the image of $f-c$ is

$$
f_{0}=y+t^{3} Q\left(t^{3} z+y p, t^{6} u-2 t^{3} z p-y p^{2}\right)-t^{2}
$$

As in the proof of Theorem 20, we apply Corollary 19 with $a=b=t^{2}$; hence, it suffices to show

$$
f_{1}=y+t^{3} Q\left(t z+(y+1) p_{1}, t^{4} u-2 t z p_{1}-(y+1) p_{1}^{2}\right)
$$

is a coordinate over $\mathbb{C}[t]$, where $p_{1}=t^{2}(y+1) u+z^{2}$. In order to apply Lemma 29, we first establish that $f_{1}$ is a strongly $t$-residual coordinate, and hence a residual coordinate over $\mathbb{C}[t]$. Clearly it is a coordinate modulo $t$; to see it is a coordinate over $\mathbb{C}\left[t, t^{-1}\right]$, define $D_{1}=t^{2}(y+1) \frac{\partial}{\partial z}-2 z \frac{\partial}{\partial u}$, and set $\phi_{1}=\left(y+t^{3} Q\left(t z, t^{4} u\right), z, u\right) \circ$ $\exp \left(\frac{p_{1}}{t^{3}} D_{1}\right)$. One may quickly check that $\phi_{1}(y)=f_{1}$.

Now, taking $\beta=t^{2}$ in Lemma 29] it suffices to show that $y+t^{3} Q\left(t z+(y+1) p_{2}, t^{2} u-2 t z p_{2}-y p_{2}^{2}\right)$ is a coordinate, where $p_{2}=(y+1) u+z^{2}$. But aside from replacing $t$ by $x$, we recognize this as $y+x^{3} Q(v, w)$ conjugated by $(y-1, z, u)$, so we are done since $y+x^{3} Q(v, w)$ is a coordinate by Theorem [13,

By Theorem 1, all Vénéreau-type polynomials are stable coordinates. However, no bound is given on the number of additional variables needed. It was previously shown by Freudenburg [6] that for $f_{1}$ and $f_{2}$, only one additional variable is needed; it turns out one is sufficient for all Vénéreau-type polynomials.

Theorem 30. Let $f=y+x Q, Q \in \mathbb{C}[x][v, w]$ be a Vénéreau-type polynomial. Then there exists $\phi \in$ $\mathrm{GA}_{4}(\mathbb{C}[x])$ with $\phi(y)=f$ and $\phi$ stably tame.

A proof of this can be found in [9], and will appear as part of a more general method in [10. We remark that in the case of the Vénéreau polynomial, this produces a different coordinate system than Freudenburg's.

\section{Acknowledgements}

The author would like to thank his advisor, David Wright, for his numerous suggestions and helpful discussions. The author would also like to thank Arno van den Essen for his feedback on an early manuscript, and in particular, pointing out lemma 18

\section{References}

[1] Shreeram S. Abhyankar and Tzuong Tsieng Moh. Embeddings of the line in the plane. J. Reine Angew. Math., 276:148-166, 1975.

[2] Teruo Asanuma. Polynomial fibre rings of algebras over Noetherian rings. Invent. Math., 87(1):101-127, 1987.

[3] Joost Berson. Polynomial coordinates and their behavior in higher dimensions. PhD thesis, University of Nijmegen, 2004.

[4] Joost Berson, Arno van den Essen, and David Wright. Stable tameness of two-dimensional polynomial automorphisms over a regular ring. Preprint, 2010, arXiv:0707.3151.

[5] Daniel Daigle and Gene Freudenburg. Families of affine fibrations. In Symmetry and spaces, volume 278 of Progr. Math., pages 35-43. Birkhäuser Boston Inc., Boston, MA, 2010.

[6] Gene Freudenburg. The Vénéreau polynomials relative to $\mathbb{C}^{*}$-fibrations and stable coordinates. In Affine Algebraic Geometry, pages 203-215. Osaka Univ. Press, Osaka, 2007.

[7] Shulim Kaliman, Stéphane Vénéreau, and Mikhail Zaidenberg. Simple birational extensions of the polynomial algebra $\mathbb{C}^{[3]}$. Trans. Amer. Math. Soc., 356(2):509-555 (electronic), 2004.

[8] Shulim Kaliman and Mikhail Zaidenberg. Vénéreau polynomials and related fiber bundles. J. Pure Appl. Algebra, 192(1-3):275-286, 2004. 
[9] Drew Lewis. Coordinates arising from affine fibrations. PhD thesis, Washington University in St. Louis, 2012.

[10] Drew Lewis. Strongly residual coordinates over $\mathbb{C}[x]$. In preparation, 2012.

[11] Peter Russell and Avinash Sathaye. On finding and cancelling variables in $k[X, Y, Z]$. J. Algebra, 57(1):151-166, 1979.

[12] Martha K. Smith. Stably tame automorphisms. J. Pure Appl. Algebra, 58(2):209-212, 1989.

[13] Masakazu Suzuki. Propriétés topologiques des polynômes de deux variables complexes, et automorphismes algébriques de l'espace $\mathbf{C}^{2}$. J. Math. Soc. Japan, 26:241-257, 1974.

[14] Arno van den Essen. Polynomial automorphisms and the Jacobian conjecture, volume 190 of Progress in Mathematics. Birkhäuser Verlag, Basel, 2000.

[15] Arno van den Essen, Stefan Maubach, and Stéphane Vénéreau. The special automorphism group of $R[t] /\left(t^{m}\right)\left[x_{1}, \ldots, x_{n}\right]$ and coordinates of a subring of $R[t]\left[x_{1}, \ldots, x_{n}\right]$. J. Pure Appl. Algebra, 210(1):141$146,2007$.

[16] Stéphane Vénéreau. Automorphismes et variables de l'anneau de polynômes $A\left[y_{1}, \ldots, y_{n}\right]$. PhD thesis, Université Grenoble I, Institut Fourier, 2001. 